\title{
PROPER MOTIONS: WHAT DYNAMICAL INFORMATION CAN WE EASILY EXTRACT FROM THEM?
}

\author{
M. WYBO and H. DEJONGHE \\ Universiteit Gent, Belgium
}

\begin{abstract}
We explore the proper motion distributions of anisotropic Plummer models, and show that information on the orbital structure can be obtained, even if no distance information is available.
\end{abstract}

We may well be at the dawn of a new era, where proper motions will become commonly available. This is already the case for data on the internal kinematics of globular clusters, but other data sets, most notably samples of populations in our own Galaxy and the Galactic Bulge, are being produced (Cudworth (1986), Minniti (this symposium)).

Clearly, proper motions are very valuable, because they involve 2 components of the velocity. On the other hand, unlike radial velocities, their magnitudes depend on the distance, which, especially in Galactic work, is hard to get. Therefore, we concentrate on two applications which are not so sensitive to an uncertain distance: data in the Galactic Bulge, and data in globulars.

The distribution of proper motions (hereafter $\mathrm{pm}$ ) is a 2-dimensional probability distribution. Is is defined at every point in the sky with coordinates $(l, b)$, and gives the probability of finding a star with proper motion components $v_{c}$ and $v_{t}$ in the point $(l, b)$. The $v_{c}$-component is directed towards the center of the system. In this contribution, we assume spherical symmetry, and it suffices to simply substitute $(l, b)$ by the projected radius $r_{p}$ (to the Galactic Center or the center of the globular).

We use the simplest dynamical equilibrium model for spherical star systems which is undoubtedly the Plummer model. We consider a one-parameter family of models, which all have the same Plummer law in the mass density, but have different anisotropic 2-integral distribution functions $F(E, L)$, and hence different orbital structures (Dejonghe 1986). This orbital structure is determined by one parameter $q$, which is related to Binney's anisotropy parameter $\beta$ by

$$
\beta=1-\frac{\sigma_{\varphi}^{2}}{\sigma_{r}^{2}}=1-\frac{\sigma_{\vartheta}^{2}}{\sigma_{r}^{2}}=\frac{q}{2} \frac{r^{2}}{1+r^{2}},
$$

in standard notations. Note that $q$ and $\beta$ have the same sign.

We consider three examples. First we have the isotropic systems, with $q=\beta=0$. This case is what is normally called a Plummer model. Then we have the radial systems, with $0<q \leq 2$, and $\beta>0$. Radial orbits are prevalent, especially in the outer parts. For $q=2$ only radial orbits are present at large radii. At last we have the tangential system, with $q<0$, and $\beta<0$. Tangential orbits are prevalent, especially in the outer parts. For $q \rightarrow-\infty$ only circular orbits are present.

To carry out the calculations, it is convenient to normalise the velocities such that the escape velocity is set to unity (the normalisation factor is of course different at every point). In addition the pm-distribution is normalised to one with the projected mass density. 
Since the distribution functions are known, one simply calculates a double integral: one over the velocity component along the line of sight and secondly through the stellar system. This can be done analytically for the isotropic case and for $q=-2 n$.

We determined the pm's by calculating all the moments of the 2-dimensional pm-distribution, then calculate the probability of finding a star at $r_{p}$ without proper motion, i. e. $\mathrm{pm}_{r_{p}}(0,0)$ (the "top value"). Assuming that the distribution can be well approximated by $\operatorname{pm}_{r_{p}}\left(v_{c}, v_{t}\right)=\sum_{i, j} a_{i j}\left(1-v_{c}^{2}-v_{t}^{2}\right)^{\alpha+i} v_{c}^{2 j}$, with $\alpha=4.5-q$ from theoretical arguments, we can determine the $a_{i j}$ by matching the moments of the approximation with the true moments and its top value.

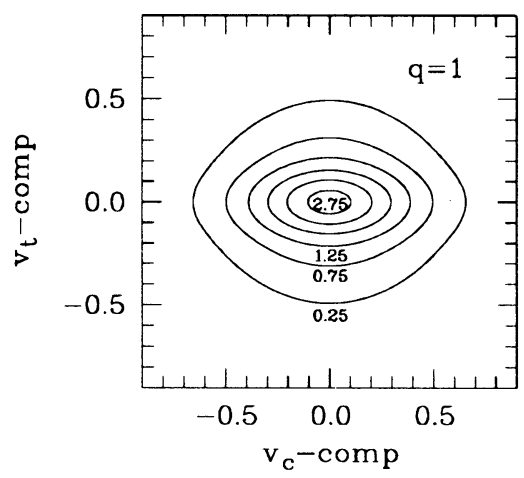

Fig.1a: Radial system

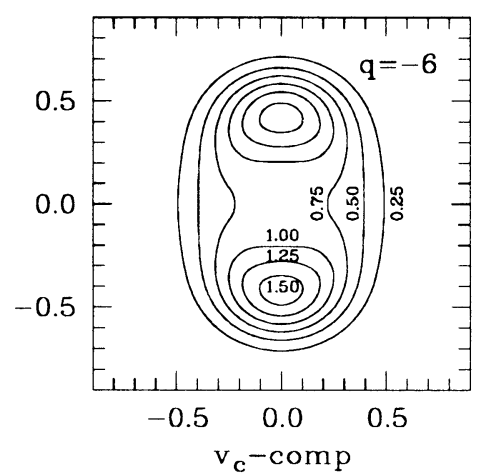

Fig.1b: Tangential system

As an illustration, we show in fig 1 the contour lines of the two-dimensional distribution, taken at a distance of 5 times the core radius. In the tangential system, the distribution function is bimodal along the $v_{t}$-axis, as can be expected due to the motion of the stars. However in the radial system the $v_{c}$ velocity-component is predominant. The isotropic case is not plotted, since the contours (trivially) are circles.

Conclusions: The distribution of proper motions provides valuable information on the orbital structure of the system, independently of the distance, by simple inspection of the contour lines. As is well known, line profiles provide important information on the kinematics of the system which is intrinsically distance independent, but the determination of the orbital structure needs inversion of the data, which has its own problems. On the other hand, comparison of the line profiles (velocity dispersions) with the velocity dispersion components of the proper motions will enable us to determine the distance to the system. However, an accuracy problem remains; we still need a lot of data to be able to determine the pm distribution accurately.

\section{References}

Dejonghe, H., 1987. Mon. Not. R. astr. Soc., 224, 13

Cudworth, 1986, $A J, 92,348$

Minniti, 1992, this symposium 